\title{
Interférences
}

Ars scribendi

$10 \mid 2018$

Varia

\section{Domitien et son ascendance herculéo-jupitérienne dans les Punica de Silius Italicus}

\section{Christophe Burgeon}

\section{(2) OpenEdition}

\section{Journals}

Édition électronique

URL : http://journals.openedition.org/interferences/5982

DOI : 10.4000/interferences.5982

ISSN : 1777-5485

Éditeur

HiSoMA - Histoire et sources des Mondes antiques

Référence électronique

Christophe Burgeon, «Domitien et son ascendance herculéo-jupitérienne dans les Punica de Silius Italicus », Interférences [En ligne], 10 | 2018, mis en ligne le 09 février 2018, consulté le 15 septembre 2020. URL : http://journals.openedition.org/interferences/5982 ; DOI : https://doi.org/10.4000/ interferences. 5982

Ce document a été généré automatiquement le 15 septembre 2020.

Tous droits réservés 


\title{
Domitien et son ascendance herculéo-jupitérienne dans les Punica de Silius Italicus
}

\author{
Christophe Burgeon
}

1 L'épopée silienne, qui voit le jour dans un contexte impérial, témoigne en partie de l'époque de son auteur, et se définit donc également par la présence d'un princeps; une présence impériale qui transparaît dans l'ensemble de la littérature romaine des premiers siècles.

2 Aux yeux de plusieurs auteurs antiques, le règne de Domitien ne doit pas être envisagé comme une réaction aux excès et à la dépravation de celui de Néron, mais plutôt comme un régime où la luxure et l'otium faisaient cause commune avec le pouvoir tyrannique. Contrairement à leurs prédécesseurs, des auteurs comme Pline ${ }^{1}$ et Juvénal ${ }^{2}$ brossent un portrait fort peu avenant de Domitien ${ }^{3}$. L'opinion qu'ils transmettent constitue le reflet, certes déformé et accentué, des sentiments que l'aristocratie a éprouvés à l'égard de l'empereur durant son règne ${ }^{4}$. Chez Juvénal, le règne de Néron est dépeint comme une «époque de sauvagerie ${ }^{5}$ ». Le satiriste nous présente par ailleurs un Domitien occupé à déchirer un monde déjà à moitié mort et un « Néron chauve » à qui Rome serait enchaînée ${ }^{6}$. Tacite, de son côté, y voit « une époque sauvage et hostile à l'excellence ${ }^{7} »$. Ces propos laissent transparaitre une ère de tyrannie durant laquelle la vertu, plutôt qu'encouragée, était rejetée. Pour certains représentants de la tradition sénatoriale, il était donc possible d'associer Domitien à Néron, ce dominus et deus contemporain de Lucain, qui faisait d'ailleurs de l'avènement de l'autocratie impériale à Rome l'un des thèmes majeurs de ses écrits.

Pourtant, à l'instar de l'Énéide, où Énée est, au fil de l'œuvre, perçu comme un protoRomain et un modèle d'inspiration pour le nouveau maitre du monde romain qu'est Auguste, Domitien apparaît sous un jour positif dans les Punica de Silius Italicus, peutêtre notamment en raison de son ascendance herculéenne et jupitérienne. 


\section{L'ascendance herculéenne de Domitien}

Tandis que Vespasien, premier princeps de la dynastie flavienne, rejetait manifestement toute association avec le fils de Jupiter et d'Alcmènes, Domitien s'efforçait effectivement d'entretenir une forte connexion avec celui-ci. Cette attitude aurait pu apparaître comme un rappel de la monarchie hellénistique et des ennemis de l'État, à l'exemple de Marc Antoine'. Une comparaison avec Hercule, qui résidait sur l'olympe, et $a$ fortiori les analogies avec Jupiter impliquaient en effet, aux yeux de ses détracteurs, une transgression de la norme sur laquelle l'empereur n'exerça bientôt plus de contrôle. Cependant, en tentant de se comparer à Hercule, Domitien faisait, entre autres, ressortir la fluidité terrifiante de la combinaison dieu/homme-bête dont le héros constituait le paradigme, et suggérait que le souverain pouvait, lui aussi, verser rapidement dans la bestialité ${ }^{10}$. En d'autres termes, si Domitien s'est approprié le modèle herculéen, il n'est pas parvenu à contrôler le glissement entre divinité et bestialité inhérent à celui-ci. Il eut toutefois le soutien de Martial ${ }^{11}$.

5 L'auteur des Punica, lui aussi, assimila l'empereur Domitien à Hercule ${ }^{12}$. Le paradigme attaché à ce modèle herculéen inspirant Domitien n'en demeure pas moins délicat, comme en témoigne la reprise de l'Hercule ambivalent de Virgile ${ }^{13}$ par les différents poètes épiques flaviens ${ }^{14}$. Dès lors, Silius compare-t-il Domitien à Hercule dans un sens laudatif?

6 Il était utile, voire nécessaire, pour un poète impérial, d'inclure çà et là dans ses vers des marques de respect envers son empereur. Domitien s'était d'ailleurs apparemment offensé de ceux qui n'avaient pas fait son éloge ${ }^{15}$. Sur la base de raisons biographiques évidentes, F. Ripoll maintient que Silius a soutenu Domitien ${ }^{16}$. W.C. McDermott et A. E. Orentzel estiment même que l'attachement de Silius à Domitien était sincère ${ }^{17}$. Pourtant, comme nous l'avons signifié précédemment, différents débats divisent les universitaires dans leur volonté de déterminer si le genre épique latin, et les Punica en l'occurrence, présentait une connotation politico-morale optimiste ou, au contraire, pessimiste. Les partisans d'une vision optimiste affirment que Silius soutenait l'idéologie impériale, soit consciemment, soit par la force des choses, et qu'il s'attachait dès lors à souligner la politique de restauration morale entreprise par les empereurs flaviens. Les adeptes d'une vision pessimiste perçoivent en revanche les Punica comme une critique de Domitien et du déclin moral et politique de Rome ${ }^{18}$. Dès lors, sommesnous en mesure de savoir si le poète soutenait ou non le régime du dernier empereur flavien? Pour répondre à cette question, nous allons nous attacher à étudier les analogies entre certaines figures des Punica et la position de Domitien.

Le statut paradigmatique de héros attribué à Hercule dans les Punica démontre clairement la vision étendue du continuum mythico-historique que possède Silius. Aux côtés de Bacchus, de Castor, de Pollux et, parfois, d'Asclépios ${ }^{19}$ et de Romulus, Hercule fait partie de ces héros divinisés pour services rendus à la race humaine ${ }^{20}$. La première position qu'occupe Hercule dans cette liste de héros suggère la primauté de son statut exemplaire. De tous ces personnages, il est effectivement le seul à être érigé en modèle à suivre. C'est sans doute la raison pour laquelle il apparaît dès le premier livre des Punica, lorsqu'il demande à la déesse Fides de venir en aide à Sagonte, une cité qu'il entend protéger. De plus, présenté comme un archétype, Hercule est imité de diverses manières par les plus grands chefs de guerre tels qu'Alexandre, Romulus, Scipion l'Africain, Pompée, César, Antoine, Auguste et Domitien. Le fait qu'il n'invite pas 
uniquement à l'imitation, mais exige également l'émulation, confère à Hercule un statut exemplaire particulièrement remarquable ${ }^{21}$.

\section{Domitien en lien avec Scipion l'Africain et Jupiter}

8 À travers une association explicite entre Scipion, d'une part, et Hercule, Bacchus et Alexandre le Grand ${ }^{22}$, d'autre part ${ }^{23}$, Silius relie l'Africain tant à des divinités qu'à un mortel brillant par sa stratégie. Faisant de Domitien le "nouveau Scipion», il se trouvait donc, lui aussi, assimilé à Alexandre, à Bacchus et à Hercule ${ }^{24}$. Conjointement, dans les Punica ${ }^{25}$, le récit apocryphe de l'ascendance jupitérienne de Scipion se mue en une réalité insistante. L'épopée laissant en outre fortement sous-entendre ${ }^{26}$ que son excellence vaudra finalement au général romain une place aux Champs Élysées situation qui n'est d'ailleurs pas sans rappeler celle du Somnium Scipionis de Cicéron -, elle établit une continuité mythico-historique entre Jupiter, Hercule, Scipion et Domitien.

Bien plus encore que son homonyme des Argonautiques ou de la Thébaïde, le Jupiter des Punica entretient des liens intimes avec les Romains, aspect que la nature historique du poème contribue en partie à expliquer. Au vers 618 du livre 3, onze vers après ceux qui ont qualifié Domitien de Germanicus, Jupiter ne parle plus de Romains, mais de «Romuléens », quand il promet que l'éloquence de Domitien surpassera un jour celle des descendants de Romulus. L'évocation de ses talents poétiques par Jupiter ${ }^{27}$ et son association à Scipion l'Africain au cours de la même théodicée contribuent à inscrire Domitien dans l'épopée, et ce, de manière indélébile. Au vers 625 du même livre, le roi de l'olympe qualifie le dernier empereur flavien de «fils des dieux" (nate deum), de la même manière que Silius, à la fin du poème, officialise la parenté divine de Scipion. Deux vers plus $\operatorname{loin}^{28}$, Jupiter promit que Quirinus en personne céderait son trône à Domitien quand celui-ci serait parvenu aux cieux. Au vers 862 du livre 13, l'apparition des termes deum gens fait, elle aussi, songer à Scipion et à Domitien, d'autant que le fantôme de la mère de Scipion, Pomponia, vient de rappeler avec insistance à son fils son ascendance divine ${ }^{29}$.

La prophétie de Jupiter constitue le passage de l'épopée qui situe le plus clairement la place occupée par la deuxième guerre punique dans le contexte de la Rome des Flaviens. Faut-il y voir une obligation panégyrique dénuée de véritable sens politique? Nous estimons que Scipion était étroitement lié au régime flavien, et qu'il servait de speculum principis à l'empereur Domitien, remplissant ainsi une fonction didactique ${ }^{30}$. C'est la raison pour laquelle Scipion, en tant qu'exemplum moral, est présenté comme un héros que Domitien se devait d'imiter.

11 Domitien apparait donc dans les Punica à la fois comme le digne successeur de Scipion l'Africain et comme le représentant terrestre de Jupiter. Silius a ainsi créé une divinité intimement proche du princeps flavien, tout en mettant en exergue un Scipion digne de susciter l'émulation et servant de guide à ce dernier.

Le portrait du Jupiter épique, tel que le brosse Silius, nous permet-il de connaître la véritable position du poète envers Domitien? Dans les Punica, le roi des dieux est dépeint en des termes parfois ambigus et alambiqués, rendant l'attitude de Silius envers son princeps difficile à décrypter. Par ailleurs, en qualifiant Rome de « royaume de Quirinus $^{31}$ ", Silius nous rappelle tant l'association implacable entre Romulus, ancêtre moral de Domitien, et sa volonté de régner seul, sans partage, que le fratricide 
qui lui est attaché : comme le premier roi de Rome, Domitien aurait tué son frère, Titus $^{32}$.

Par la même occasion, Silius fait allusion au royaume de Scipion l'Africain et à son nom quasi royal ${ }^{33}$. Cependant, l'Africain n'a jamais envisagé de régner sans partage. En outre, le livre 3 des Punica contient un panégyrique relativement long consacré à Jupiter. Celui-ci concerne la vertu qu'il perçoit chez Vespasien et ses fils, et évoque, bien sûr, l'épisode de la théodicée dans l'Énéide ${ }^{34}$, durant lequel le Jupiter de Virgile annonce les futurs exploits de la lignée julienne ${ }^{35}$. Aux vers 595-596, le roi des dieux promet par ailleurs à Vénus qu'une "race de guerriers" (bellatrix gens), à savoir les Flaviens, «fera grandir la renommée des Juliens sacrés" (Flauii, sacris augebit nomen Iulis). En termes d'exemplarité, les exploits des Flaviens dépasseront même ceux d'Auguste, dont le nom sacré surpassait déjà le superlatif Maximus ${ }^{36}$. Aux vers 594-595 du livre 3, il est même dit que "l'excellence céleste ${ }^{37}$ ", incarnée par la dynastie flavienne, parviendra jusqu'aux étoiles. Silius divinise donc Domitien.

L'affinité dont témoigne Scipion, le républicain, envers un empereur autocratique et extravagant laisse-t-elle apparaître une brèche nettement apparente sur le plan idéologique ? Pour les héros de l'épopée silienne, en découle la possibilité d'émerger en tant qu'exemplum de domination individuelle sur la destinée romaine. Toutefois, d'après nous, Silius présente Domitien selon un schéma post-lucainien de la guerre civile, qui lui est propre et est loin d'être résolument négatif. Cette ambiguïté sur la personne du dernier empereur flavien, caractéristique de la période, n'empêche donc pas Silius de présenter Domitien sous un angle globalement positif, en plaçant la vertu romaine au premier plan, et en lui faisant remporter un certain nombre de victoires authentiques. En effet, alors que Valerius ne célèbre que l'activité poétique de l'empereur ${ }^{38}$, Silius développe longuement ses campagnes militaires ${ }^{39}$.

Qu'en est-il de ces expéditions militaires? Quand le Jupiter de Silius évoque le triomphe de Domitien face aux Chattes en $83^{40}$, et mentionne son titre de Germanicus ${ }^{41}$, peut-être convient-il de considérer cette héroïsation comme un trait ironique. En faisant allusion à la peur que le jeune Domitien inspirait aux Bataui ${ }^{42}$, Jupiter nous livre un panégyrique de la jeunesse de l'empereur qu'il aligne ainsi sur l'héroïsme fougueux du Scipion des Punica $^{43}$. Toutefois, il n'est pas impossible que cette dernière référence puisse, elle aussi, être malicieuse si on la confronte avec le récit donné par Tacite ${ }^{44}$, selon lequel les Bataui se rendirent avant même que Domitien ne soit parvenu sur le champ de bataille ${ }^{45}$. Il en va de même quand Jupiter attribue de la témérité à Domitien ${ }^{46}$ pour avoir trouvé refuge dans le temple capitolin au moment de l'assaut des partisans de Vitellius, en $69^{47}$. Ici encore, la version que Tacite ${ }^{48}$ nous donne de Domitien en train de se cacher laisse place à une tout autre interprétation de la gesta impériale. Au surplus, le vers $686 \mathrm{du}$ livre 14 des Punica $^{49}$ aurait été rédigé pour mettre en valeur le rôle pacificateur de Domitien $^{50}$. Cependant, nous inclinons à penser qu'en mentionnant ces épisodes Silius rappelle non seulement la participation de l'empereur à des affrontements - qu'il juge héroïques - contre un ennemi extérieur, mais aussi sa capacité à se préserver pour pouvoir véhiculer, et ce, le plus longtemps possible, les valeurs romaines ancestrales.

On le voit, Silius cherche, semble-t-il, à nous rappeler que Scipion l'Africain, fils de Jupiter, ne pouvait que montrer l'exemple, et ouvrir la voie aux empereurs désireux de 
garantir les valeurs traditionnelles romaines tout en n'abusant pas de leur pouvoir. Tout parallèle entre cette figure républicaine et Domitien témoigne d'un désir de dresser le portrait d'un exemplum moral dont les actes, globalement positifs et moralement louables, faisaient écho à ceux du Flavien.

Le poète flavien se tenait tantôt entre tradition et innovation, tantôt entre moralité républicaine - à son apogée au lendemain de la victoire du vertueux Scipion l'Africain sur Hannibal en 202 apr. J.-C. -, et morale flavienne. Tout en jetant des ponts entre passé et présent, et en mettant en garde son lecteur contre les dangers inhérents aux guerres civiles (il fut très certainement bousculé par les événements qui ont jalonné l'« année des quatre empereurs »), pour des raisons idéologiques, il y a lieu de penser qu'il subordonna son républicanisme à sa vision pro-domitienne. Les individus mis en scène dans les Punica se font d'ailleurs le miroir des débats tant républicains qu'impériaux; Scipion l'Africain, le plus vertueux d'entre tous, et Domitien étaient placés sur une même ligne de conduite et d'action. Tout cela contribue à voir en Scipion un princeps idéal, dont Domitien est incité à suivre l'exemple ${ }^{51}$. Corrélativement, nous estimons que les Punica doivent être considérés comme la représentation de l'évolution morale graduelle de Rome depuis l'ère post-augustéenne, où les valeurs traditionnelles étaient en péril, jusqu'à la fin de l'ère flavienne, durant laquelle ces mêmes valeurs, selon Silius, furent revitalisées.

La mise en valeur de Romains exemplaires par Silius génère diverses questions concernant leur qualité même d'exemple. L'adhésion à ce type de représentation livienne - conduit à s'interroger sur le statut de modèle des personnages ainsi dépeints, de même que sur le processus par lequel la poésie épique établit les archétypes historiques ${ }^{52}$. Dans ses Punica, Silius décrit un passé idéal contrastant avec le déclin qui lui succède. De temps à autre, il laisse apparaître un comportement romain exemplaire qui conserve son intégrité tout au long de l'histoire. Ainsi Scipion et Domitien sont deux des parangons romains dignes d'aemulatio.

\section{BIBLIOGRAPHIE}

ANDERSON A. R. 1928, « Heracles and His Successors: A Study of a Heroic Ideal and the Recurrence of a Heroic Type », HSPh 39, p. 7-11.

BARTSCH S. 1994, Actors in the Audience. Theatricality and Doublespeak from Nero to Hadrian, Revealing Antiquity 6, Cambridge, Harvard University Press.

BERNSTEIN N. W. 2008, In the Image of the Ancestors. Narratives of Kingship in Flavian Epic, Phoenix. Suppl. 48, Toronto, University of Toronto Press.

BRAUN L. 1993, « Der Aufbau der Punica des Silius Italicus », WJA 19, p. 173-183.

ELTER A. 1907, Donarem Pateras... (Horat. Carm. 4, 8), II, 1, Bonn, C. Georg.

GALINSKY G. K. 1972, The Herakles Theme. The Adaptations of the Hero in Literature from Homer to the Twentieth Century, Oxford, Basil Blackwell. 
HARDIE P. 1993, The Epic Successors of Virgil. A Study in the Dynamics of a Tradition, Roman Literature and Its Contexts, Cambridge, Cambridge University Press.

HENDERSON J. 1997, Figuring Out Roman Nobility. Juvenal's Eighth Satire, Exeter Studies in History, Exeter, University of Exeter Press.

JONES B. W. 1992, The Emperor Domitian, Londres, Routledge.

MCDERMOTT W. C., ORENTZEL A. E. 1977, « Silius Italicus and Domitian », AJPh 98, p. 32-38.

MCGUIRE D. T. 1985, History as Epic: Silius Italicus and the Second Punic War, Ph. D. Cornell University.

MALAmUd M., MCGUiRE D. T. 1993, « Flavian Variant: Myth Valerius, Argonautica », in A. J. BoYLE (ed.), Roman Epic, Londres, Routledge, p. 212-218.

MARKS R. 2005, From Republic to Empire: Scipio Africanus in the Punica of Silius Italicus, Studien zur klassischen Philologie 152, Francfort-sur-le-Main, P. Lang.

MORGAN L. 1997, « Achilleae Comae: Hair and Heroism According to Domitian », CQ 47, p. 209-214.

RAMAGE E. S. 1989, « Juvenal and the Establishment. Denigration of Predecessor in the 'Satires' », ANRW, II, 33, p. 640-707.

RIPOLL F. 1998, La morale héroïque dans les épopées latines d'époque flavienne. Tradition et innovation, Bibliothèque d'études classiques 14 , Louvain, Peeters.

TIPPING B. 2010, Exemplary Epic. Silius Italicus' Punica, Oxford Classical Monographs, Oxford, Oxford University Press.

ZECCHINI G. 2011, « Silio Italico e Domiziano », in L. CASTAGNA, G. GALIMBERTI BIFFINo, C. RIBoldi (a cura di.), Studi su Silio Italico, Milan, V\&P, p. 28-34.

\section{NOTES}

1. Pline le Jeune, Pan. 2.

2. Juvénal, 4, 150-151.

3. RAMAGE 1989 ; JONES 1992, p. 160 ; BRAUN 1996, p. 269-275.

4. JONES 1992.

5. Juvénal, 4, 148-149: illa [...] tempora saeuitiae.

6. Juvénal, 4, 37-38. Voir MORGAN 1997.

7. Tacite, Ag. 1, 4 : saeua et infesta uirtutibus tempora.

8. Suétone, Ves. 12.

9. MAlAmUd, MCGUIRE 1993, p. 212 ; Suétone, Aug. 50 ; Pline l'Ancien, Nat. 35, 92-94.

10. HARDIE 1993, p. 67-68.

11. JONES 1992, p. 32.

12. ELTER 1907, p. 40 et 75 ; ANDERSON 1928, p. 7-8 et 44-58; GALINSKY 1972, p. 140-149; HARDIE 1993, p. 67-68.

13. Virgile, A. 8, 190-305.

14. Stace, Theb. 1, 470-520 ; Verrius Flaccus, 2, 373-578.

15. Dion Cassius, LXVII, 4, 2 ; Pline le Jeune, Pan. 1, 6. 
16. RIPOLL 1998, p. 469-471.

17. MCDERMOTT, ORENTZEL 1977, p. 34.

18. D. T. McGuire fait partie des pessimistes.

19. Bacchus, Castor, Pollux et Asclépios ne figurent pas dans les Punica.

20. Cicéron, Leg. 2, 19 ; Tusc. 1, 27-28 ; N.D. 2, 62 ; Horace, Carm. 3, 3, 9-36 ; 4, 8, 13-34 ; Ep. $2,1,5-11$.

21. TIPPING 2010, p. 16 ; GALINSKY 1972, p. 5.

22. Face à la brièveté de la vie, Alexandre encourage Scipion à poursuivre le destin victorieux prédit par son père divin Jupiter dans l'audace et la promptitude (Silius Italicus, 13, 762-775). Voir aussi BALDWIN 1990, p. 51-60.

23. Silius Italicus, $15,78-81 ; 647-650$.

24. MARKS 2005, p. 222-223 ; BARTSCH 1994, p. 148-187.

25. Silius Italicus, 4, 476; 7, 487-488; 13, 615-649; 17, 653-654. Voir MARKS 2005, p. 187-203 ; BERNSTEIN 2008, p. 150.

26. Silius Italicus, $15,77-78 ; 17,645-654$.

27. Silius Italicus, 3, 618-621: Quin et Romuleos superabit uoce nepotes / quis erit eloquio partum decus. Huic sua Musae / sacra ferent, meliorque lyra, cui substitit Hebrus / et uenit Rhodope, Phoebo miranda loquetur.

28. Silius Italicus, 3, 627-628: solioque Quirinus / concedet, mediumque parens fraterque locabunt.

29. Silius Italicus, $13,628-647$.

30. MARKS 2005, p. 211-244.

31. Silius Italicus, 6, 103 : sceptra Quirini.

32. Une certaine tradition anti-domitienne a prétendu que Domitien avait empoisonné Titus (Dion Cassius, LXVI, 26; Philostrate, VA, VI, 32 ; Hérodien, IV, 5, 6 ; Aurelius Victor, Ep. 10-11 ; peut-être aussi Chants sibyllins, XII, 121-123), ce qui n'est nullement avéré dans la mesure où ce dernier mourut à Aquae Cutiliae, station thermale située à l'est de Rieti, de fièvre (Suétone, Tit. 10 ; Dom. 2 ; Eusèbe de Césarée, Chron., année 2096) ou de l'abus de bains (Plutarque, De sanitate praecepta 3). Pourtant, étonnamment, Titus était encore en vie quand Domitien partit à Rome à cheval pour se rendre au camp des prétoriens afin de s'y faire saluer en tant qu'empereur et d'y distribuer une somme égale à celle donnée par son frère au lendemain de la mort de Vespasien (Suétone, Dom. 2 ; Tit. 11 ; Dion Cassius, LXVI, 26).

33. HENDERSON 1997, p. 142 ; MARKS 2005, p. 206.

34. Virgile, A. 1, 257-296.

35. Virgile, A. 1, 278-296.

36. HARDIE 1993, p. 5.

37. Silius Italicus, 3, 594 : uirtus caelestis.

38. Verrius Flaccus, 1, 12-15.

39. Silius Italicus, 3, 606-617.

40. MARKS 2005, p. 220.

41. Silius Italicus, 3, 607 : at tu transcendes, Germanice, facta tuorum. 
42. Silius Italicus, 3, 608 : iam puer auricomo praeformidate Batauo.

43. MARKS 2005, p. 220-222.

44. Tacite, Hist., IV, 85, 6.

45. MCGUIRE 1985, p. 178-179.

46. Silius Italicus, 3, 609-610: nec te terruerint Tarpei culminis ignes:/ sacrilegas inter flammas seruabere terris.

47. MARKS 2005, p. 220.

48. Tacite, Hist. 3, 69-74.

49. Silius Italicus, 14,686 : uiri qui nunc dedit otia mundo.

50. ZECCHINI 2011, p. 33.

51. MARKS 2005, p. 287.

52. TIPPING 2010, p. 9.

\section{RÉSUMÉS}

The sylvian epic, which originated in an imperial context, partly reflects the era of its author, and is therefore also defined by the presence of a princeps; an imperial presence which is reflected in the whole of Roman literature of the first centuries. In the eyes of many ancient writers, Domitian's reign should not be seen as a reaction to the excesses and depravity of Nero's, but rather as a regime in which lust and otium coexist with tyrannical power. Like the Aeneid, where Aeneas is, throughout the work, perceived as a proto-Roman and a model of inspiration for the new master of the Roman world, Augustus, Domitian appears in a positive light in the Punica de Silius Italicus, perhaps mainly because of his Herculean and Jupiterian descent. The Flavian poet seeks, it seems, to remind us that Scipio Africanus, son of Jupiter, could only lead by example, and open the way for emperors willing to guarantee traditional Roman values while not abusing not their power. Any parallel between this republican figure and Domitian testifies to a desire to paint a portrait of a moral exemplum whose acts, generally positive and morally commendable, echoed those of the Flavian.

\section{INDEX}

Index géographique : Rome

oeuvrecitee Chants sibyllins

nomsmotscles Aurelius Victor, Cicéron, Dion Cassius, Eusèbe de Césarée, Hérodien, Homère, Horace, Juvénal, Martial, Philostrate l'Athénien, Pline le Jeune, Plutarque, Silius Italicus, Stace, Suétone, Tacite, Valerius Flaccus (Gaius), Virgile

Keywords : Hercules, Domitian

Mots-clés : Hercule, Domitien 
AUTEURS

CHRISTOPHE BURGEON

Université catholique de Louvain 